03,05

\title{
Примесная магнитная восприимчивость полупроводников в случае прямого обменного взаимодействия в модели Изинга
}

\author{
() Н.А. Богословский, П.В. Петров, Н.С. Аверкиев \\ Физико-технический институт им. А.Ф. Иоффре, \\ Санкт-Петербург, Россия \\ E-mail: nikitabogoslovskiy@gmail.com
}

Поступила в Редакцию 25 июня 2019 г.

В окончательной редакции 25 июня 2019 г.

Принята к публикации 2 июля 2019 г.

\begin{abstract}
Посредством численного моделирования исследована зависимость примесной магнитной восприимчивости от температуры. Учитывалось прямое обменное взаимодействие магнитных моментов случайно распределенных в пространстве примесей в рамках модели Изинга. При понижении температуры зависимость магнитной восприимчивости в такой системе перестает соответствовать закону Кюри, что связано с образованием фазы спинового стекла. Показано, что вид предэкспоненциального множителя в формуле для прямого обменного взаимодействия существенно влияет на температурную зависимость магнитной восприимчивости только в случае ферромагнитного обмена.
\end{abstract}

Ключевые слова: легированные полупроводники, спиновое стекло.

DOI: $10.21883 /$ FTT.2019.11.48404.538

\section{1. Введение}

Магнитная восприимчивость вещества определяется величиной и количеством магнитных моментов, видом взаимодействия между моментами, так что исследование восприимчивости дает информацию о магнитной структуре материала. К настоящему времени по данной тематике накоплен огромный массив как экспериментальных данных, так и теоретических расчетов, в частности, магнитная восприимчивость примесных полупроводников активно исследуется уже более тридцати лет [1]. Интерес к теме магнитных свойств полупроводников значительно вырос после открытия ферромагнетизма в кристаллах GaAs легированных магнитной примесью марганца [2,3], Теоретическая модель Зенера, предложенная для описания ферромагнитного состояния в легированных полупроводниках [4], предсказывала возможность получения ферромагнитных материалов на основе $\mathrm{GaN}$ и $\mathrm{ZnO}$ с температурой Кюри выше комнатной, что весьма привлекательно с точки зрения разработки новых видов полупроводниковых приборов. Однако, за прошедшее время, несмотря на значительные усилия технологов, получены образцы, температура Кюри в которых лишь немного превышает $100 \mathrm{~K}$, что ставит под сомнение теоретические выводы работы [4]. В связи с вышесказанным, исследование магнитных свойств легированных полупроводников обусловненных взаимодействием примесных атомов представляется весьма актуальным.

Существует значительное разнообразие типов магнитной структуры, обусловленное как видом взаимодействия между магнитными моментами вещества, так и геометрической упорядоченностью их расположения. Наличие дальнего порядка в расположении магнитных моментов приводит к формированию ферро- и антиферромагнитных фаз в зависимости от знака обменного взаимодействия. В материалах с отсутствием дальнего порядка при низких температурах как правило возникает фаза спинового стекла, характеризуемая наличием множества близких по энергии состояний с различной конфигурацией магнитных моментов. Эти энергетические состояния разделены потенциальными барьерами, вследствие чего для спиновых стекол характерны медленные релаксационные процессы. Спиновые стекла активно изучаются на примере сплавов магнитных и немагнитных металлов, например меди и марганца [5]. Свойства спиновых стекол на основе легированных полупроводников изучены значительно слабее. Основным отличием таких материалов является другой вид обменного взаимодействия. В металлах магнитные моменты вещества взаимодействуют косвенным образом, через посредство свободных электронов (РККИ-взаимодействие). Для такого типа обмена характерна знакопеременная зависимость от расстояния $r$ между магнитными моментами, которая спадает степенным образом как $r^{-3}$.

В полупроводниках существенными являются прямой обмен между примесными атомами, а также взаимодействие примесных атомов как магнитных диполей. Дипольное взаимодействие в полупроводниковых спиновых стеклах проявляется значительно слабее, так как оно эффективно усредняется по направлениям. Поэтому существенным для формирования стекольного состояния оно является только в случае очень низких концентраций и температур [6]. В обычных условиях основым фактором определяющим магнитные свойства легированных полупроводников является зависимость прямого обменного взаимодействия от расстояния между магнитными моментами. Отличительной особенностью легированных полупроводников от других физических систем является неупорядоченное расположение 
примесных центров. В изучениии подобных объектов хорошо зарекомендовали себя численные методики $[7,8]$. В настоящей работе мы численно, с помощью алгоритма Метрополиса исследуем зависимость магнитной восприимчивости от температуры для ферро- и антиферромагнитного случаев.

При исследовании таких систем принято записывать энергию обменного взаимодействия между примесными атомами в виде $J \sim \exp (-2 r / a)$, где $a-$ боровский радиус примесного состояния. При этом, обычно, пренебрегается наличием предэкспоненциального множителя степенным образом зависящего от $r$. Известно, что для водородоподобной модели предэкспоненциальный множитель имеет показатель степени равный 5/2 [9,10]. Для примесных состояний в твердых телах показатель степени может отличаться от водородоподобной модели, например для глубоких донорных состояний он равен -1 [11]. В нашей работе показано, что показатель степени $r$ для ферромагнитного типа взаимодействия существенным образом влияет на ход зависимости магнитной восприимчивости от температуры. Им определяется наличие или отсутствие характерного максимума (cusp) восприимчивости при переходе в состояние спинового стекла. Для антиферромагнитного взаимодействия влияние предэкспоненциального множителя значительно слабее.

К настоящему моменту примесная магнитная восприимчивость мелких примесных центров в полупроводниках хорошо исследована как теоретически, так и экспериментально [1,12]. Однако, вопрос о взаимодействии более сложно устроенных примесных атомов, таких например как магнитная примесь марганца в материалах $\mathrm{A}^{3} \mathrm{~B}^{5}$, или многозарядных примесных центров до сих пор не освещался. Результаты наших расчетов могут быть использованы для интерпретации измерений примесной магнитной восприимчивости в материалах с такими примесями и использованы для исследования их межпримесного обменного взаимодействия.

\section{2. Численное моделирование}

При моделировании рассматривался кубический объем полупроводника с циклическими граничными условиями, в котором случайным образом распределялись магнитные примеси. Количество примесных атомов в наших расчетах, как правило, было равно 10000, что связано с ограничениями по объему памяти. Мы использовали модель Изинга, согласно которой каждый примесный атом обладает магнитным моментом, проекция которого $\sigma$ может быть направлена по или против оси $O z$. Соответствующий гамильтониан называют гамильтонианом Изинга.

$$
H=\sum_{i>j} J\left(r_{i j}\right) \sigma_{i} \sigma_{j} .
$$

Здесь суммирование ведется по всем парам атомов $(i, j)$. Мы учитываем взаимодействие всех примесных атомов между собой, при этом энергия взаимодействия зависит от расстояния между атомами $r_{i j}$, при расчетах мы рассматривали различные виды зависимости энергии от расстояния $J\left(r_{i j}\right)$. Положительным значениям $J\left(r_{i j}\right)$ соответствует случай антиферромагнитного взаимодействия, а отрицательным - ферромагнитного. Мы исследовали поведение магнитной восприимчивости для обменного взаимодействия вида

$$
J\left(r_{i j}\right)=\left(r_{i j} / a\right)^{n} e^{-2 r_{i j} / a} .
$$

Здесь $a-$ боровский радиус носителя заряда на примесном атоме. Существенным для задачи является значение параметра $\mathrm{Na}^{3}$, где $N$ - концентрация магнитных моментов. Используемый нами вид зависимости величины обменного взаимодействия от расстояния был получен в приближении больших расстояний между примесными атомами $r \gg a$, что соответствует случаю $N a^{3} \ll 1$ [9-11]. В наших расчетах среднее расстояние между примесными центрами составляло $N^{-1 / 3}=4.5 a$, что соответствует значению $\mathrm{Na}^{3}=0.01$.

Далее для рассматриваемой системы запускалась процедура термализации при помощи алгоритма Метрополиса. Мы рассматривали случайно выбранный атом и считали изменение энергии $\Delta E$ системы при изменении знака магнитного момента. В случае, если переворот магнитного момента приводит к уменьшению энергии системы, мы принимаем данную перестановку. В случае, когда переворот магнитного момента приводит к увеличению энергии, мы производим переворот с вероятностью $\exp (-\Delta E / k T)$. Термализация проводилась до тех пор, пока энергия системы не начинала флуктуировать около некоторого среднего значения. Известно, что по величине этих флуктуаций можно найти многие параметры системы, например, из флуктуаций энергии можно рассчитать теплоемкость, а из флуктуаций намагниченности системы $M$ можно рассчитать магнитную восприимчивость $\chi$. Действительно, магнитная восприимчивость вещества определяется как

$$
\chi=\frac{\partial M}{\partial H},
$$

где $H-$ магнитное поле. Используя распределение Гиббса, намагниченность системы $M$ можно выразить через намагниченность $M_{i}$ и энергию $E_{i}$ отдельных микросостояний как

$$
M=\frac{\Sigma M_{i} \exp \left(-E_{i} / k T\right)}{\Sigma \exp \left(-E_{i} / k T\right)} .
$$

Записывая энергию как $E_{i}=-M_{i} H+C_{i}$, где $C_{i}$ - независящее от $H$ слагаемое описывающее взаимодействие магнитных моментов, легко найти магнитную восприимчивость:

$$
\begin{aligned}
\frac{\partial M}{\partial H}= & \frac{\Sigma M_{i}^{2} / k T \exp \left(-E_{i} / k T\right)}{\Sigma \exp \left(-E_{i} / k T\right)} \\
& -\frac{\Sigma M_{i} \exp \left(-E_{i} k T\right) \Sigma M_{i} / k T \exp \left(-E_{i} / k T\right)}{\left(\Sigma \exp \left(-E_{i} / k T\right)\right)^{2}} .
\end{aligned}
$$




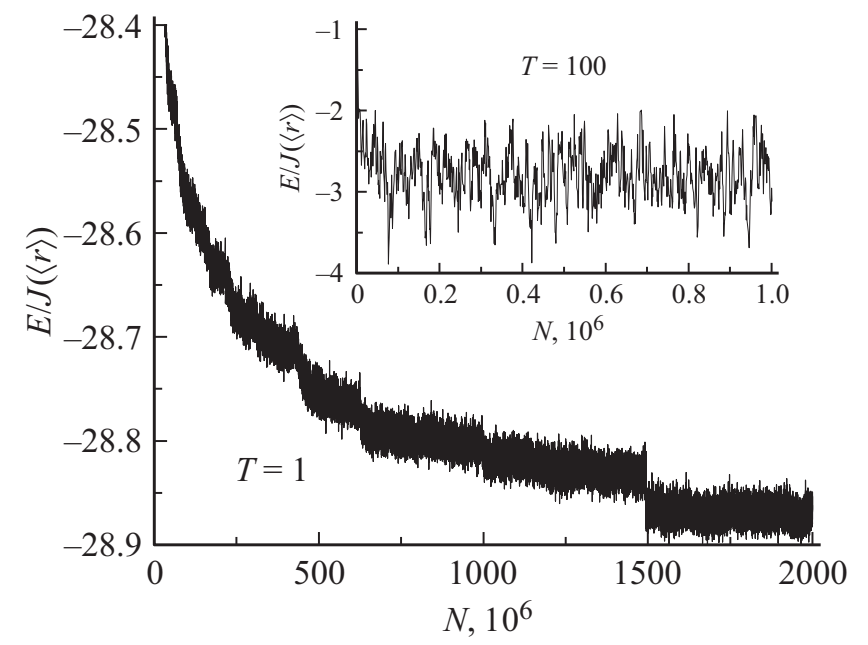

Рис. 1. Энергия взаимодействия системы магнитных примесей в пересчете на один центр в зависимости от номера шага $N$ в алгоритме Метрополиса. Температура указана на графике в единицах магнитного взаимодействия на среднем расстоянии между примесями.

Отсюда непосредственно следует, что

$$
\chi=\frac{\left\langle M^{2}\right\rangle-\langle M\rangle^{2}}{k T} .
$$

Результаты численного расчета магнитной восприимчивости через флуктуации магнитного момента усреднялись по некоторому числу шагов. Этот расчет повторялся для различных реализаций случайного распределения в пространстве магнитных атомов. Число шагов моделирования необходимое для приемлимого усреднения сильно зависело от температуры. На рис. 1 показана зависимость энергии взаимодействия в системе магнитных примесей от номера шага для случая ферромагнитного взаимодействия. Энергия и температура на графике нормированы на энергию взаимодействия на среднем расстоянии. Для высоких температур сисистема очень быстро, примерно за $10^{5}$ шагов, релаксировала к некоторому среднему значению, около которого продолжала флуктуировать. При температурах сравнимых с энергией взаимодействия на среднем расстоянии релаксация энергии в системе значительно замедлялась. Требовалось совершить не менее $10^{9}$ шагов для вычисления низкотемпературного значения восприимчивости. Это связано с переходом системы в фазу спинового стекла и свойственными ей медленными релаксационными процессами.

\section{3. Обсуждение результатов}

На рис. 2 и 3 приведены зависимости магнитной восприимчивости от температуры для антиферромагнитного и ферромагнитного случаев и разных значений показателя степени предэкспоненциального множителя $r^{n}$. При высоких температурах ход зависимости соответствует закону Кюри $\chi \sim T^{-1}$. Взаимодействие магнитных моментов приводит к возникновению ближнего порядка в системе и отклонению магнитной восприимчивости от закона Кюри с понижением температуры. При этом поведение для ферромагнитного и антиферромагнитного случаев отличается. В случае антиферромагнитного взаимодействия ход восприимчивости слабо зависит от показателя степени предэкспоненты $n$, тогда как для ферромагнитного взаимодействия при увеличении $n$ проявляется так называемый „сusp“ максимум на температурной зависимости при температурах примерно соответствующих энергии взаимодействия магнитных моментов на среднем расстоянии. Наличие этого максимума свидетельствует о переходе отдельных примесных кластеров в состояние ферромагнитных доменов. В случае однородного ферромагнетика при температурах близких к температуре Кюри $T_{c}$

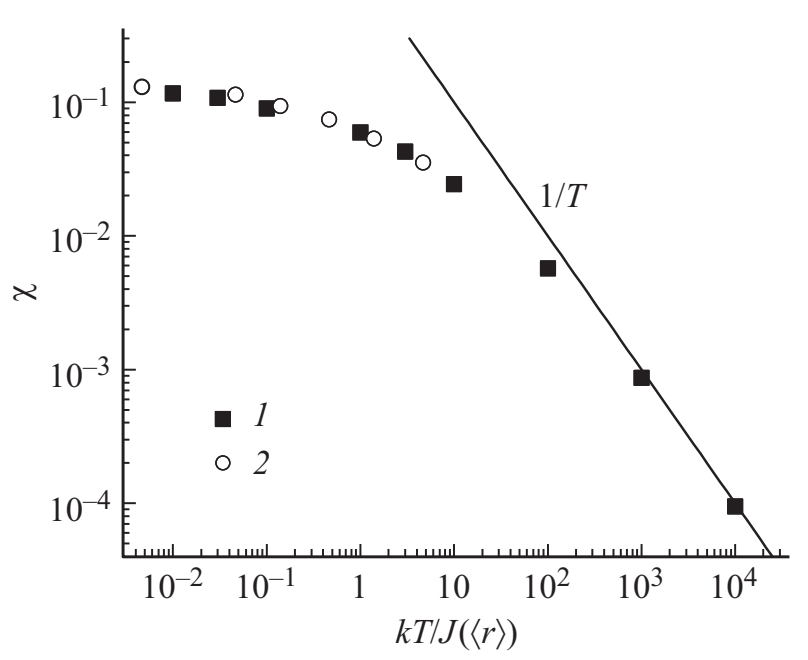

Рис. 2. Зависимость магнитной восприимчивости от температуры для антиферромагнитного взаимодействия примесей. Вид предэкспоненциального множителя: $1-r^{5}, 2-1$.



Рис. 3. Зависимость магнитной восприимчивости от температуры для ферромагнитного взаимодействия примесей. Вид предэкспоненциального множителя: $1-r^{5}, 2-r^{5 / 2}, 3-100$, $4-1,5-r^{-1}$. 


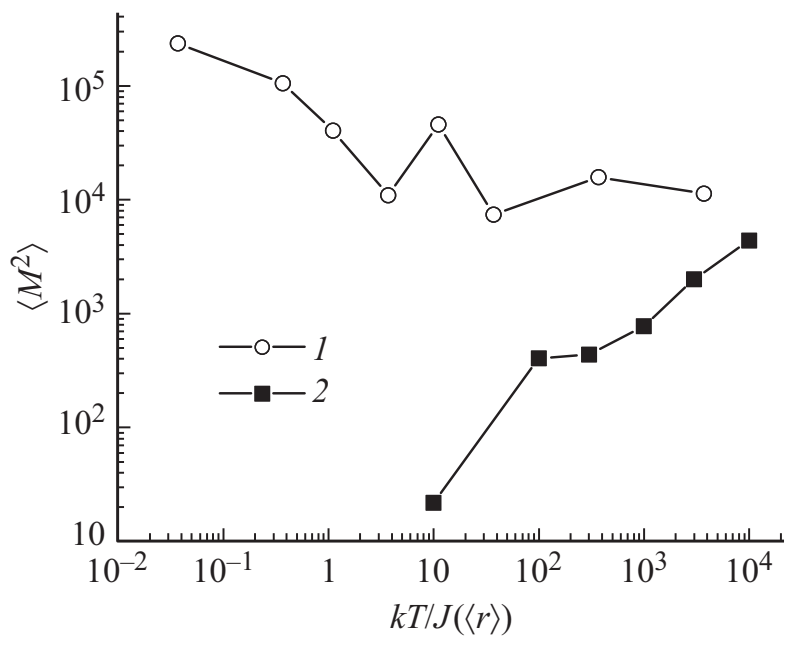

Рис. 4. Зависимость среднеквадратичного магнитного момента от температуры для ферромагнитного и антиферромагнитного взаимодействия примесей. Вид предэкспоненциального множителя: $1-r^{5}, 2--r^{-1}$.

восприимчивость расходится как $\left(T-T_{c}\right)^{-1}$. Это происходит из-за гигантских флуктуаций магнитного момента при переходах между состояниями с противоположно ориентированными магнитными доменами. В системе неупорядоченных магнитных моментов температура перехода в ферромагнитное состояние неоднородна по объему из-за флуктуаций концентрации примесных центров. Поэтому расходимость на температурной зависимости размывается в широкий пик небольшой амплитуды. При высоких показателях степени предэкспоненциального множителя „сusp“ выражен более ярко, что связано с наличием некоторой оптимальной энергии взаимодействия между магнитными моментами которой соответствует определенная температура перехода. Оптимальная энергия должна соответствовать максимуму произведения функции $J(r)$ на вероятность найти примесный атом на расстоянии $r$ которая пропорциональна квадрату расстояния. Функция $r^{2} r^{n} \exp (-2 r / a)$ имеет максимум при $r=(n+2) a / 2$. Тогда температура при которой должен наблюдаться максимум магнитной восприимчивости, измеряемая в единицах $J(\langle r\rangle)$, определяется выражением $J((n+2) a / 2) / J(\langle r\rangle)$. Для показателей степени $n=5 / 2$ и $n=5$ это выражение дает температуры $T \approx 16 J(\langle r\rangle)$ и $T \approx 3 J(\langle r\rangle)$, что хорошо согласуется с результатами численных расчетов приведенных на рис. 3 .

В наших расчетах для ферромагнитного знака взаимодействия всегда возникала хаотически намагниченная доменная структура, то есть средний магнитный момент системы был близок к нулю, как и для антиферромагнитного знака. Однако, температурная зависимость среднего квадрата магнитного момента для этих двух случаев сильно различается, как это видно из рис. 4. Для случая ферромагнитного взаимодействия среднеквадратичная намагниченность растет, то есть домены при понижении температуры увеличиваются в размерах. Для антиферромагнитного взаимодействия картина противоположна, флуктуации намагниченности стремятся к нулю, то есть антиферромагнитный порядок устанавливается на все более малых расстояних.

\section{4. Выводы}

Таким образом, нами было численно исследовано поведение примесной магнитной восприимчивости в легированных полупроводниках в зависимости от температуры для межпримесного обменного взаимодействия вида $J(r)= \pm(r / a)^{n} \exp (-2 r / a)$. Показано, что при низких температурах, независимо от знака взаимодействия, закон Кюри для примесной магнитной восприимчивости перестает работать, что связано с образованием фазы спинового стекла. При этом в случае ферромагнитного межпримесного взаимодействия наблюдалось нетривиальное поведение восприимчивости при изменении показателя степени предэкспоненциального множителя функции $J(r)$. На температурной зависимости магнитной восприимчивости появлялся максимум связанный с тем, что при определенных значениях степени предэкспоненциального множителя существует оптимальная энергия межпримесного обменного взаимодействия. Совпадение температуры и этой энергии значительно увеличивает флуктуации магнитного момента в системе и способствует появлению максимума восприимчивости в зависимости от температуры.

\section{Финансирование работы}

Работа поддержана грантом РФФИ № 19-02-00283.

\section{Конфликт интересов}

Авторы заявляют об отсутствии конфликта интересов.

\section{Список литературы}

[1] M.P. Sarachik, D.R. He, W. Li, M. Levi, J.S. Brooks. Phys. Rev. B 31, 1469 (1985).

[2] H. Ohno, A. Shen, F. Matsukura, A. Oiwa, A. Endo, S. Katsumoto, Y. Iye. Appl. Phys. Lett. 69, 363 (1996).

[3] A.H. MacDonald, P. Schiffer, N. Samarth. Nature Mater. 4, 195 (2005).

[4] T. Dietl, H. Ohno, F. Matsukura, J. Cibert, D. Ferrand. Science 287, 1019 (2000).

[5] O. Zhai, D.C. Harrison, D. Tennant, E.D. Dahlberg, G.G. Kenning, R.L. Orbach. Phys. Rev. B 95, 054304 (2017).

[6] F. Holtzberg, J.L. Tholence, H. Godfrin, R. Tournier. J. Appl. Phys. 50, 1717 (1979).

[7] N.A. Bogoslovskiy, P.V. Petrov, Y.L. Ivánov, K.D. Tsendin, N.S. Averkiev. Phys. Rev. B 98, 075209 (2018).

[8] N.A. Bogoslovskiy, P.V. Petrov, N.S. Averkiev. Temperat. Phys. 45, 146 (2019).

[9] Л.П. Горьков, Л.П. Питаевский. Докл. АН 151, 822 (1963).

[10] C. Herring, M. Flicker. Phys. Rev. 134, A362 (1964).

[11] Н.С. Аверкиев, С.Ю. Ильинский. ФТТ 36, 503 (1994).

[12] C.T. Murayama, W.G. Clark, J. Sanny. Phys. Rev. B 29, 6063 (1984).

Редактор Т.Н. Василевская 